\title{
Footprint Calculation Methods for a Reusable Launch Vehicle
}

\author{
Anhtuan D. Ngo and David B. Doman \\ U. S. Air Force Research Laboratory (AFRL/VACA) \\ 2210 Eighth St., Bldg. 146 \\ Wright-Patterson AFB, OH. 45433-7531
}

\begin{abstract}
An important element of an adaptive and reconfigurable guidance and control system is the ability to compute the largest area (footprint) on the earth's surface that is reachable by the autonomous vehicle under the current operating condition. During an abort situation when it suddenly becomes necessary to divert the vehicle from its original landing site, the knowledge of this reachable area can assist in choosing a safe and available landing site. In this paper, a technique based on calculus of variations is applied to find the footprint of an autonomous vehicle subject to operating constraints.
\end{abstract}

\section{Introduction}

Over the past decade, increasing effort on achieving affordable and reliable access to space has produced a number of advances in propulsion, structures, materials as well as system guidance and control for reusable launch vehicles. In the areas of guidance and control a variety of methods to enhance the safety and operability of these autonomous hypersonic vehicles have been proposed with promising results [1], [2], [3]. These methods enable the vehicle to adapt and reconfigure under off-nominal conditions that are the result of control failures or damage. Because of the high cost penalty for additional weights, these autonomous hypersonic vehicles do not have much hardware redundancy. As a result, a single control actuator failure can severely affect the vehicle's performance and safety. Control actuator failures therefore along with their effects on mission performance and safety must be considered in the whole process of trajectory planning and retargetting. An important element of the vehicle's safe operation in such contingent flying conditions is the ability to compute in real time the largest reachable area on the earth 's surface given its current conditions, constraints on skin temperature, structural load, and achievable aerodynamics. The reachable area is referred as the footprint of the vehicle. The footprint information is useful in the event of a system failure and the flight path of the vehicle needs to be altered. In this paper, we extend the footprint computation methods suggested by Schultz [4] and Vinh [5] to enable one to compute footprints for vehicles that have experienced control effector failures or are experiencing off-nominal operating conditions.

\section{Problem Formulation}

An unforseen failure in the vehicle system, such as a malfunctioning in the propulsion or hydraulic system, during the ascent or reentry phases of the flight can make it necessary to divert the vehicle from its intended landing site. With the propulsion system unavailable during the contingency, the descent trajectory planning and guidance then consist of an entry phase and a terminal area energy management phase. The landing site for the unpowered flight vehicle is chosen according to the largest area on the earth's surface reachable by the vehicle.

Assuming that the vehicle angular orientation is maintained by its inner loop attitude controller, the motion of the unpowered vehicle over a non-rotating earth can be modelled as a point mass:

$$
\begin{aligned}
\dot{h} & =v \sin (\gamma) \\
\dot{\theta} & =v \cos (\gamma) \cos (\psi) \\
\dot{\phi} & =v \cos (\gamma) \sin (\psi) \\
\dot{v} & =\frac{-D}{m}-\frac{\mu \sin (\gamma)}{\left(R_{o}+h\right)^{2}} \\
\dot{\gamma} & =\frac{L \cos (\sigma)}{m v}-\frac{\mu \cos (\gamma)}{v\left(R_{o}+h\right)^{2}}+\frac{v \cos (\gamma)}{\left(R_{o}+h\right)} \\
\dot{\psi} & =\frac{L \sin (\sigma)}{m v \cos (\gamma)}-\frac{v \cos (\gamma) \cos (\psi) \tan (\phi)}{\left(R_{o}+h\right)}
\end{aligned}
$$

Where

$$
h \text { : Altitude }
$$




$$
\begin{aligned}
\theta & : \text { Longitude } \\
\phi & : \text { Latitude } \\
v & : \text { Velocity } \\
\gamma & : \text { Flight path angle } \\
\psi & : \text { Heading angle } \\
m & : \text { Mass of the vehicle } \\
R_{o} & : \text { Earth's Radius } \\
\mu & : \text { Gravitational Parameter }
\end{aligned}
$$

The total energy of the unpowered vehicle is strictly decreasing because of the non-conservative forces acting on the vehicle ,most notably, the aerodynamic drag. The vehicle footprint then consists of points on the earth' surface at which the total energy decreases to a set value. When its energy reaches this value, the vehicle then enters the final part of its trajectory called terminal area energy management phase. Combining its velocity $v$ and altitude $h$ in an energy-state approximation [6], a reduced order model can be obtained to simplify the vehicle description. The specific energy of the vehicle can be expressed as

$$
E=\frac{1}{2} v^{2}+\frac{\mu}{\left(R_{o}+h\right)^{2}} h
$$

From Equations 1, 4, and 7, one can derive an expression for the time rate of change in the vehicle's energy:

$$
\frac{d E}{d t}=-\frac{v D}{m}-\frac{2 \mu h v \sin (\gamma)}{\left(R_{o}+h\right)^{3}}<0
$$

Assuming that the vertical forces acting on the vehicle are in equilibrium and its vertical motion frequency dynamics are much faster its horizontal motion dynamics, the flight path angle can be taken to be near zero, i.e., $\gamma \approx 0$. Moreover, since the acceleration normal to the velocity vector is small, i.e., $(v \dot{\gamma} \approx 0)$ and $v \neq 0$, the time-rate of change of the flight path angle can then be taken to be essentially zero, i.e., $\dot{\gamma} \approx 0$. From Equation 5 , we have

$$
L=\frac{m}{\cos (\sigma)}\left(\frac{\mu}{\left(R_{o}+h\right)^{2}}-\frac{v^{2}}{\left(R_{o}+h\right)}\right)
$$

With

$$
\begin{gathered}
L=\frac{1}{2} \rho v^{2} S_{a} C_{L} \\
D=\frac{1}{2} \rho v^{2} S_{a} C_{D}, \text { and } C_{D}=C_{D_{o}}+k C_{L}^{2}
\end{gathered}
$$

we can combine Equations 9 and 10 into Equation 11 to have

$$
D=\frac{1}{2} \rho v^{2} S_{a} C_{D_{o}}+\frac{2 m^{2} k \tilde{g}^{2}}{\rho v^{2} S_{a} \cos ^{2} \sigma}
$$

where $\tilde{g}$ is the effective acceleration due to gravity (nominal gravitational acceleration with centripetal relief effects):

$$
\tilde{g}=\frac{\mu}{R_{o}+h}-\frac{v^{2}}{R_{o}+h}
$$

Given the initial velocity $v\left(t_{o}\right)=v_{o}$, and initial altitude $h\left(t_{o}\right)=h_{o}$, the vehicle specific energy $E(t)$ can be calculated according Equation 8:

$$
E(t)=E_{o}+d E=\frac{1}{2} v_{o}^{2}+\frac{\mu h_{o}}{\left(R_{o}+h\right)^{2}}+d E
$$

The vehicle's velocity $v(t)$ can be derived from the current specific energy $E(t)$ :

$$
v(t)=\sqrt{2 E(t)-\frac{\mu h}{\left(R_{o}+h\right)^{2}}} \approx \sqrt{2 E(t)}
$$

since the vehicle flight altitude $h$ is small when compared to the earth's radius $\boldsymbol{R}_{\boldsymbol{o}}$. From the above discussion, the unpowered vehicle under energy-state approximations has the reduced-order model $\dot{x}=f(x, u, t)$ of the form:

$$
\begin{aligned}
\dot{\theta} & =\frac{v \cos (\psi)}{\left(R_{o}+h\right) \cos (\phi)} \\
\dot{\phi} & =\frac{v \sin (\psi)}{R_{o}+h} \\
\dot{\psi} & =\frac{z \tilde{g}}{v}-\frac{v \cos (\psi) \tan (\phi)}{R_{o}+h} \\
\dot{E} & =\frac{-D v}{m}
\end{aligned}
$$

where $x=[\theta, \phi, \psi, E]^{T}$ is the vehicle state vector. The control inputs $u=\left[\begin{array}{ll}\rho & z\end{array}\right]^{T}$ are the air density, $\rho=\rho(h)$, and the tangent of the bank angle, $z=\tan (\sigma)$. The optimization objective is to find the control vector $u=\left[\begin{array}{ll}\rho & z\end{array}\right]$ such that, at time $t=t_{f}$, the crossrange position specified by the vehicle latitude $\phi\left(t_{f}\right)$ is maximized for a given downrange value $\theta_{f}=\theta\left(t_{f}\right)$ :

$$
\max _{\rho, z} J=\int_{t_{o}}^{t_{f}} G d t=\int_{t_{o}}^{t_{f}}-\dot{\phi} d t
$$

The initial values of the vehicle states are taken to be $\theta\left(t_{o}\right)=0, \phi\left(t_{o}\right)=0, \psi\left(t_{o}\right)=0$ and $E\left(t_{o}\right)=E_{o}$.

The Hamiltonian function $H$ to be minimized is

$$
\begin{aligned}
H & =G+\lambda^{T} f \\
& =-\dot{\phi}+\lambda_{\theta} \dot{\theta}+\lambda_{\phi} \dot{\phi}+\lambda_{\psi} \dot{\psi}+\lambda_{E} \dot{E}
\end{aligned}
$$

The weighting coefficients $\lambda$ are the Lagrangian multipliers to be determined. The necessary conditions for optimality are

$$
\begin{aligned}
& \frac{\partial H}{\partial x}=-\frac{d \lambda}{d t} \\
& \frac{\partial H}{\partial u}=0
\end{aligned}
$$

Taking variations of the Hamiltonian function $H$, enforcing the condition $\partial H=0$ at the stationary point of 
$H$, and the Lagrangian multipliers do not vary at the boundary produces

$$
\left[H \delta t-\lambda^{T} \delta\right]_{t_{o}}^{t_{f}}=0
$$

The final heading $\psi\left(t_{f}\right)$, the final down-range position $\theta\left(t_{f}\right)$ are unspecified. The initial and final values for the Lagrangian multipliers $\lambda\left(t_{o}\right)$ are chosen to be zero. The dynamics of $\lambda(t)$ for $t_{o}<t<t_{f}$ can be found from Equations 22:

$$
\begin{aligned}
& \dot{\lambda}_{\psi}=-\frac{\partial H}{\partial \psi}, \quad \dot{\lambda}_{E}=-\frac{\partial H}{\partial E} \\
& \dot{\lambda}_{\theta}=-\frac{\partial H}{\partial \theta}, \dot{\lambda}_{\phi}=-\frac{\partial H}{\partial \phi}
\end{aligned}
$$

Combining with Equation 18, it can be shown that

$$
\begin{aligned}
\lambda_{\theta}(t) & =0 \\
\lambda_{\phi}(t) & =1-\cos \left(\theta_{f}-\theta\right) \\
\lambda_{\psi}(t) & =-\cos (\phi) \sin \left(\theta_{f}-\theta\right) \\
\lambda_{E}(t) & =\frac{H_{r}}{\dot{E}}=\frac{\lambda_{\theta} \dot{\theta}+\lambda_{\phi} \dot{\phi}+\lambda_{\psi} \dot{\psi}}{\dot{E}}
\end{aligned}
$$

At the optimal control $u_{\text {opt }}, H\left(x, u_{\text {opt }}, \lambda, t\right)=0$ and

$$
H\left(x, u_{o p t}, \lambda, t\right) \leq H\left(x, u_{o p t}+\Delta u, \lambda, t\right)
$$

that is,

$$
\begin{aligned}
& H_{r}\left(x, u_{o p t}, \lambda, t\right)+\lambda_{E} \dot{E}\left(x, u_{o p t}, \lambda, t\right) \leq \\
& H_{r}\left(x, u_{o p t}+\Delta u, \lambda, t\right)+\lambda_{E} \dot{E}\left(x, u_{o p t}+\Delta u, \lambda, t\right) .
\end{aligned}
$$

From Equations 27 and 28, it can be shown that Substituting

$$
\lambda_{E}(t)=\frac{H_{r}\left(x, u_{o p t}, \lambda, t\right)}{\dot{E}\left(x, u_{o p t}, t\right)}
$$

into equation 29 , we have

$$
\frac{H_{r}\left(x, u_{o p t}, \lambda, t\right)}{\dot{E}\left(x, u_{o p t}, \lambda, t\right)}-\frac{H_{r}\left(x, u_{o p t}+\Delta u, \lambda, t\right)}{\dot{E}\left(x, u_{o p t}+\Delta u, \lambda, t\right)} \leq 0
$$

From Expression 30, we can define a new Hamiltonian function that relates the vehicle states $x$, its co-states $\lambda$, control $u$ to its decreasing rate of energy $\dot{E}$ :

$$
H_{\text {new }}=\frac{H_{r}}{\dot{E}}=\frac{-\dot{\phi}+\lambda_{\theta} \dot{\theta}+\lambda_{\phi} \dot{\phi}+\lambda_{\psi} \dot{\psi}}{\dot{E}}
$$

Substituting values for the state rates $\dot{\phi}, \dot{\theta}, \dot{\psi}, \dot{E}$ and their co-states $\lambda_{\phi}, \lambda_{\theta}, \lambda_{\psi}$ into Equation 31 , we obtain:

$$
H_{n e w}=\frac{\left[\begin{array}{l}
\frac{-v \sin (\psi)}{R_{o}+h}+\lambda_{\phi} \frac{-v \sin (\psi)}{R_{o}+h} \\
+\lambda_{\psi}\left[z \left(\frac{\mu}{v\left(R_{o}+h\right)^{2}}-\frac{v}{R_{o}+h}-\frac{v \cos (\psi) \tan (\phi)}{R_{o}+h}\right.\right.
\end{array}\right]}{-v\left(\frac{.5 v^{2} S_{a} C_{d_{o}} \rho}{m}+\frac{2 m k \tilde{g}^{2}\left(1+z^{2}\right)}{\rho S_{a} v^{2}}\right)}
$$

For a decreasing total energy $(\dot{E}<0)$, the new Hamiltonian $H_{\text {new }}$ is to be maximized with the control parameters being the air density $\rho$ and the bank angle $\sigma$. For a hypersonic vehicle under energy-state approximations, methods suggested by Schultz [4] and Vinh [5] are applied. Since the control parameter $\rho$ does not appear in the numerator of $H_{\text {new }}, H_{\text {new }}$ is maximized when the air density $\rho$ is minimized. From the drag Equation 11, we have

$$
\frac{\partial D}{\partial \rho}=0 \Rightarrow \rho_{o p t}=\sqrt{\frac{4 m^{2} k \tilde{g}^{2}\left(1+z^{2}\right)}{v^{2} S_{a} C_{d o}}}
$$

The optimum bank angle $\sigma$ associated with $z=\tan (\sigma)$ is

$$
\frac{\partial H}{\partial z}=0 \Rightarrow z_{o p t}=\frac{\cos (\phi) \sin (\tilde{\theta})\left(\frac{\mu}{R^{2}}-\frac{v^{2}}{R}\right) \frac{R}{v^{2}}}{\cos (\tilde{\theta}) \sin (\psi)-\sin (\phi) \sin (\tilde{\theta}) \cos (\psi)}
$$

with $\tilde{\theta}=\theta_{f}-\theta, R=R_{o}+h$. From Equations 33 and 34 the final downrange $\theta_{f}=\theta\left(t_{f}\right)$ of the vehicle is needed apriori to generate an optimal bank angle $\phi_{\text {opt }}$ and altitude $\rho_{\text {opt }}=\rho(h)$ to achieve the largest final crossrange $\phi_{f}=\phi\left(t_{f}\right)$. To generate the vehicle entire footprint, it is suggested in [5] to use the vehicle heading $\psi_{o}=\psi\left(t_{o}\right)$ as the sweeping parameters: $0^{\circ} \leq \psi_{o} \leq 90^{\circ}$. The intermediate downrange $\theta_{f}^{\prime}$ and crossrange $\phi_{f}^{\prime}$ are transformed by the coordinate transformation to obtain the final $\theta_{f}$ and $\phi_{f}$ :

$$
\begin{aligned}
\tan \left(\theta_{f}\right) & =\tan \left(\theta_{f}^{\prime}\right) \cos \left(\psi_{o}^{\prime}\right)+\frac{\tan \left(\phi_{f}^{\prime}\right) \sin \left(\psi_{f}^{\prime}\right)}{\cos \left(\theta_{f}^{\prime}\right)} \\
\sin \left(\phi_{f}\right) & =\sin \left(\phi_{f}^{\prime}\right) \cos \left(\psi_{o}^{\prime}\right)-\sin \left(\theta_{f}^{\prime}\right) \cos \left(\phi_{f}^{\prime}\right) \sin \left(\psi_{f}^{\prime}\right)
\end{aligned}
$$

Equations 33 and 34 give the optimal crossrange $\phi\left(t_{f}\right)$ for a given downrange $\theta_{f}$. Iterations on the initial values of $\theta_{f}$ may be necessary so that the initial guess matches the final value $\theta\left(t_{f}\right)$ resulting from the Equations 18 .

\section{Approximate Solution}

Online footprint computation using the above method may be slow because iterations on $\theta_{f}$ are necessary. An approximate solution to the maximum crossrange calculation was proposed by Vinh [5] for the optimal bank angle command signal.

$$
z_{\text {approx }}=\tan \left(\frac{1}{2} e^{\kappa} \arctan \left(\frac{\cos (\phi)}{\tan (\psi)}\right)\right)
$$

where

$$
\kappa=\frac{1}{10 \sqrt{k C_{d o}}}
$$

The advantage of this approximate solution is that it does not require an initial guess of $\theta\left(t_{f}\right)$ and subsequent iterations. This bank angle control was demonstrated in [5] with good results. 


\section{Application to a Reusable Launch Vehicle}

In this section, we apply the methods for the footprint calculation to the $\mathrm{X}-33$ reusable launch vehicle. The $\mathrm{X}-33$ is an autonomous, reusable launch vehicle that has two linear aerospike engines and eight aerodynamic control surfaces: inner/outer elevons, rudders, flaps as shown in Figure 1. At the beginning of the reentry phase, the 2500 -slug vehicle attains the velocity of 10,000 feet/second at an altitude of 180,000 feet. With this initial energy, the unpowered vehicle's crossrange is calculated until its final energy is equivalent to the energy at the final speed of 2500 feet/second and 50,000 feet of altitude. The air density constraint which is related to thermal constraint on the vehicle is $\rho<7.397 \times 10^{-5}$ slugs $/ \mathrm{ft}^{3}$. Similarly, structurerelated air density constraint has an upper limit of $3.981 \times 10^{-5}$ slugs $/ \mathrm{ft}^{3}$. An important requirement that

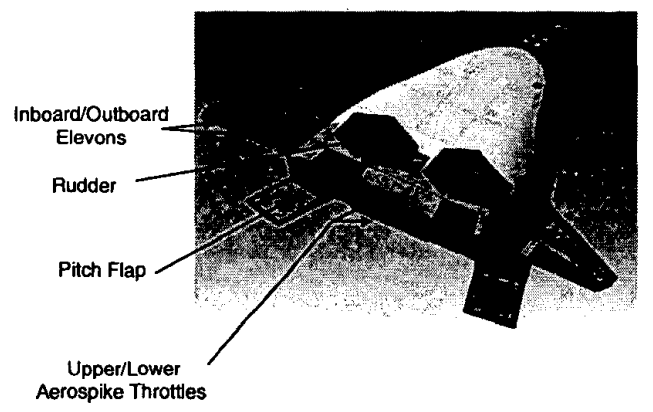

Figure 1: X-33 Reusable Launch Vehicle

must be satisfied in calculating the vehicle footprint is the maintenance of lift to effective-weight equilibrium $(\mathrm{L}=\mathrm{W})$ while banking the vehicle:

$$
L=\frac{1}{2} \rho^{2} S_{a} C_{L}=W=\frac{m \tilde{g}}{\cos (\sigma)}
$$

where $S_{a}$ is vehicle planform area and $\rho$ is the air density. The respective normal and axial force coefficients $C_{N}$ and $C_{A}$ obtained from the vehicle's aerodynamic table are transformed into the corresponding lift and drag coefficients $C_{L}$ and $C_{D}$ :

$$
\begin{aligned}
& C_{L}=\cos (\alpha) C_{N}-\sin (\alpha) C_{A} \\
& C_{D}=\sin (\alpha) C_{N}+\cos (\alpha) C_{A}
\end{aligned}
$$

A root solver based on the Secant method is then used to minimize the residual of $L-W$. As discussed earlier, the new Hamiltonian $H_{n e w}$ in Equation 31 is optimized when the vehicle's drag is minimized. Scanning the altitude that is 20,000 feet above and below the vehicle's current altitude, we look for the next optimal altitude command that minimizes the vehicle's drag subject to the constraint $L=W$. Once the optimal altitude is found, the bank angle command $\sigma$ is calculated using Vinh's control law in Equation 35. As shown in Equation 35, the bank angle command is a function of the vehicle's current parasitic drag $C_{D_{o}}$ coefficient and induced drag parameter $k$. The parasitic drag coefficient $C_{D_{0}}$ in Equation 11 for the X-33 vehicle is found from the aerodynamic data table by iterating over the vehicle's angle of attack $\alpha$ at a given Mach number to find the drag coefficient at zero lift:

$$
C_{D}=\left.C_{D_{o}}\right|_{C_{L}(\alpha, M a c h)=0}
$$

A third-order polynomial expression is then used to parameterize $C_{D_{\mathrm{o}}}$ in terms of the vehicle's velocity to give the following Equation:

$$
\begin{aligned}
C_{D_{o}}= & -2.8809 \times 10^{-4} \mathrm{Mach}^{3}+7.4321 \times 10^{-3} \mathrm{Mach}^{2} \\
& -6.0914 \times 10^{-2} \mathrm{Mach}+2.9697 \times 10^{-1}
\end{aligned}
$$

The parameter $k$ in Equation 11 can be found by looking up the values of the lift and drag coefficients from the vehicle's aerodynamic data table and solving:

$$
k=\frac{C_{D}-C_{D_{\circ}}}{C_{L}^{2}}
$$

\section{Rotational Equilibrium Under Failures}

In addition to ensuring that the lift on the vehicle equals its weight, rotational equilibrium must also be enforced to maintain the vehicle's attitude. A trim routine is used to find the aero-control positions that are necessary to balance the base pitching moment produced by the wing-body portion of the vehicle. The lateral directional moments resulting from the wingbody portion of the vehicle are assumed to be zero ( i.e. an assumption of zero steady-state sideslip and wing-body symmetry). In the trim routine, the roll, pitch and yaw control effectiveness of each aero-control surface at a fixed Mach number and angle of attack is found from the aerodynamic table using small perturbations. With $B$ being the pitch control effectiveness matrix, $\delta$ the aero-control deflections and $M_{o}$ the base pitching moment, a linear programming formulation [3] is used to find $\delta$ such that:

$$
\begin{gathered}
\min _{\delta} J=\left\|B \delta-M_{o}\right\|_{1} \\
\text { subject to } \quad \underline{\delta} \leq \delta \leq \bar{\delta}
\end{gathered}
$$

where $\underline{\delta}$ and $\bar{\delta}$ are vectors that represent the lower and upper limits of the control effectors. Control surface failures such as locked control surfaces are accommodated by setting the upper and lower limits for the locked effectors equal to one another. By solving the minimization problem posed in Equation 36, the potentially undesirable moments produced by locked effectors are automatically taken out by the un-failed 
surfaces whenever it is physically possible to do so. One additional comment regarding Equation 36 is in order. The linear programming problem that is posed, assumes that the moments are linearly related to surface deflections. This is rarely the case in practice and at best, at a particular flight condition, the moments are nonlinear functions of surface deflection and in order to find the deflections that produce a desired moment one must find $\delta$ that solves the following equation subject to $\underline{\delta} \leq \delta \leq \bar{\delta}$

$$
M_{o}=f(\delta)
$$

In general, if the solution to Equation 36 which we will call $\delta_{l p}$ is substituted for $\delta$ into Equation 37 one will find that:

$$
M_{o} \neq f\left(\delta_{l p}\right)
$$

An iterative approach has been developed here that solves multiple linear programming problems. The approach finds a modified value of base pitching moment, namely $M_{o_{\text {mod }}}$ that produces a control deflection vector $\delta_{l p_{\text {mod }}}$ that produces

$$
M_{o}=f\left(\delta_{l p_{\text {mod }}}\right)
$$

The iterative procedure uses the following update rule to compute $M_{o_{\text {mod }}}$.

$$
M_{o_{\text {mod }_{k+1}}}=M_{o_{\text {mod }_{k}}}+\omega\left(M_{o}-f\left(\delta_{l p_{\text {mod }_{k}}}\right)\right)
$$

where $k$ indicates the $k$ th iteration, and $\omega$ is a parameter that affects the convergence properties. As stated previously, a locked control surface can be accommodated directly through the control allocator by setting the upper and lower position limits equal to one another. This ensures that the remaining control surfaces are used to balance the possibly undesirable effects of the locked surfaces as well as balance the wing-body moments. In most cases, one or more locked surfaces create a condition that requires the free surfaces to move to off-nominal positions to counter undesirable effects of the locked surfaces to maintain rotational equilibrium. This ultimately translates into force perturbations that increase the overall drag on the vehicle and therefore reduces the size of the vehicle footprint.

\section{Example: Locked Surfaces}

The X-33 aerodynamic model is used in this example to obtain representative force and moment data for an RLV. The case under consideration uses the techniques described above to compute footprints under nominal conditions and in the case of right inboard and outboard elevons both locked at -30 degrees. In order to maintain rotational equilibrium, the control allocator automatically deflects the left inboard and outboard elevons to -30 degrees to balance the lateral directional moments and deflects the body flaps to balance the pitching moments produced by the other control surfaces and the wing-body. This trimming operation is performed at each time step at which the equations of motion and control commands are computed, in this case, the integration time step is 1 second. Figure 2 compares the footprints of the nominal and failed vehicle and one can see that the failed vehicle footprint is reduced in size. This is because of the increase in drag due to the off-nominal control surface deflections that are required to maintain rotational equilibrium.

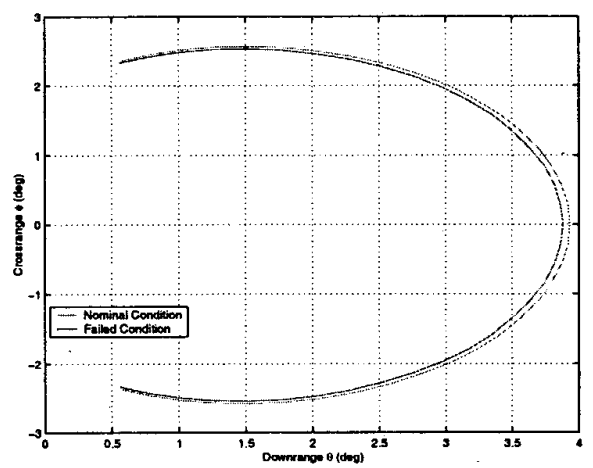

Figure 2: Footprint Comparison Between Nominal and Failed Conditions with Right Inboard and Outboard Elevons Locked at $(-30 \mathrm{deg})$

Figure 3 shows the commanded altitude that yields minimum drag from the initial energy to the final energy to yield the largest crossrange . The discrete profile of commanded altitude arises from the increments used in the altitude sweep. A smoother time history can be obtained by using smaller altitude increments or using results from the coarse sweep to initialize an numerical optimizer to find the altitude at which minimum drag occurs. Using the sweeping method to initial a numerical optimizer reduces the likelihood of the getting trapped at a local minimum. Both approaches result in increased computational time. Figure 4 shows the time history of the bank angle command to achieve the maximum crossrange. The entire footprint of the vehicle is then generated by setting the initial heading $\psi\left(t_{o}\right)$ from 90 degrees to 0 degree and calculating the vehicle trajectory given the initial and final energies.

\section{Effect of Maximum Trimmable Angle of Attack}

It should be noted that control surface failures can also reduce the range of trimmable angles of attack on some vehicles. This can cause a maximum wing-body lift coefficient constraint to be replaced by a more conser- 


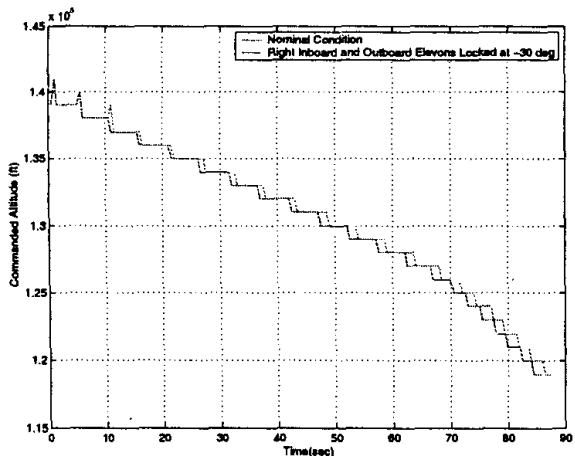

Figure 3: Altitude Command for the Maximum Crossrange

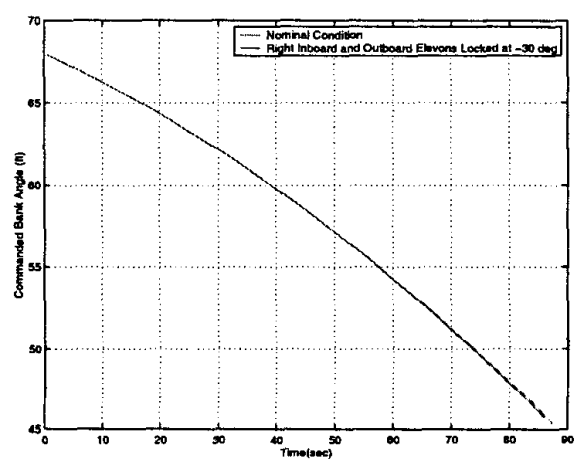

Figure 4: Bank Angle for the Maximum Crossrange

vative maximum trimmable lift coefficient constraint. Whether such constraints become active or not is dependent upon the vehicle; however, loss or damage to any of the aero-control surfaces could potentially reduce the maximum trimmable angle of attack. This reduction can decrease the maximum achievable lift that strongly influences the cross-range that can be achieved by banking the vehicle. In the case studied here, such a constraint was never active because the $\mathrm{X}-33$ requires less control power to trim at high angles of attack than at low angles of attack. Additionally, minimum drag conditions on this vehicle generally occur at high altitude and at high angles of attack. Nevertheless, it is interesting to look at the reduction in the size of the vehicle footprint that would result from a hypothetical reduction in trimmable angle of attack. Such a case can be seen in Figure 5. In calculating the two footprints shown in Figure 5, the vehicle initial conditions remains the same while its maximum trimmable angle of attack is reduced from 50 degrees to 7 degrees.

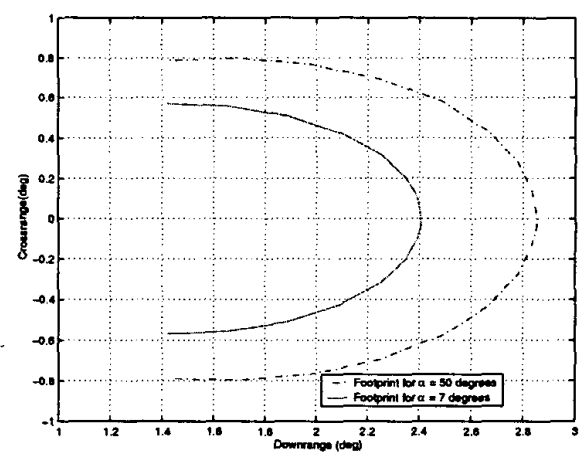

Figure 5: Footprint Sensitivity to Varying Constraints on Maximum Angle of Attack

\section{Conclusion}

Methods are presented that allow one to estimate the area on the earth's surface that can be reached by a reusable launch vehicle experiencing control effector failures. The optimal method is based on variational calculus and requires iterations on the initial guess. The suboptimal method is based on approximations but more practical for implementation. A numerical example is used to show how the vehicle's largest reachable area can shrink under failure. Such information is required to select landing sites under failure conditions.

\section{References}

[1] E. N. Johnson, A. J. Calise and J. E. Corban, "Adaptive Guidance and Control for Autonomous Launch Vehicles," Proceedings of the IEEE Aerospace Conference, Big Sky, MT March 2001.

[2] J. D. Schierman, D. G. Ward, J. F. Monaco and J. Hull, "Adaptive Guidance Systems for Hypersonic Vehicles with Inner-Loop Reconfigurable Control," Proceedings of the IEEE Aerospace Conference, Big Sky, MT March 2001.

[3] D. B. Doman and A. D. Ngo, "Dynamic Inversion-Based Adaptive/Reconfigurable Control of the X-33 on Ascent," Journal of Guidance, Control, and Dynamics, Vol. 25, No. 2, 2002.

[4] R. L. Schultz, "Three-Dimensional Trajectory Optimization for Aircraft," Journal of Guidance, Control and Dynamics, Vol. 13, No. 6, November December 1990, pp. 936-943.

[5] N. X. Vinh, "Optimal Trajectories in Atmospheric Flight," Elsevier Scientific Publishing Company.

[6] E. S. Rutowski, "Energy Approach to the General Aircraft Performance Problem," Journal of Aeronautical Sciences Vol. 21, 1954, pp. 187-195. 